\title{
Synthesis and Antibacterial Screening of Novel Fluorine Containing Heterocycles
}

\author{
B.K. KARALE ${ }^{1 *}$, S.J. TAKATE ${ }^{2}$, S.P. SALVE², B.H. ZAWARE² and S.S. JADHAV ${ }^{2}$ \\ 'Department of Chemistry, Radhabai Kale Mahila Mahavidyalya, Ahmednagar - 414001, India. \\ 2Department of Chemistry, New Arts, Commerce and Science College, Ahmednagar - 414001, India. \\ *Corresponding author E-mail: bkkarale@yahoo.com \\ http://dx.doi.org/10.13005/ojc/310135
}

(Received: January 01, 2015; Accepted: February 11, 2015)

\section{ABSTRACT}

\begin{abstract}
A Claisen-Schimidt condensation of 2-hydroxyacetophenones 1a-f with an aldehyde 2 yielded a series of compounds 3a-f. Compounds 3a-f were transformed into series of substituted chromones 4a-f, pyrazolines 5a-f and aurones 6a-f on treatment with DMSO/ $\mathrm{I}_{2}, \mathrm{NH}_{2} \mathrm{NH}_{2} / \mathrm{EtOH}$ and $\mathrm{Hg}(\mathrm{OAc})_{2} /$ pyridine respectively. All these novel compounds were characterized by spectral techniques. All the compounds were screened for their antibacterial potential.
\end{abstract}

Key words: Pyrazoles, Flavonoids, Pyrazolines, Aurones, Fluorine.

\section{INTRODUCTION}

Although the progress of science is rapid, the treatment of infectious diseases is still a major challenge for scientific world. This is mainly because of the increasing microbial infections and mutations occurring in microbial strains which make them resistant to many of the current therapeutic agents. The main approach to overcome this problem is designing and developing efficient drug molecules. In search of new pharmaceuticals various heterocycles have been investigated for their antimicrobial potential. Recent literature survey showed that azoles especially fluorinated one are dominating in this field. Among the various azoles pyrazoles, $\mathrm{N}$-substituted pyrazoles and other heterocycles with pyrazole moiety have emerged as potential bioactive compounds ${ }^{1-4}$.

Thiazoles are another important members of azole family known for their various biological properties such as antioxidant ${ }^{5}$, antimicrobial ${ }^{6-8}$, anticancer ${ }^{9}$ and analgesic ${ }^{10}$ etc. More recently it has been shown that thiazole containing pyrazoles have proved their potential as antimicrobial ${ }^{11,12}$ agents. Chalcones are open chain flavonoids widely distributed in plants and are known to possess diverse biological properties like antimicrobial ${ }^{13}$, antitubercular ${ }^{14,15}$, antioxidant ${ }^{15}$ and antitumor ${ }^{16}$. More recently pyrazolyl chalcones have been 
studied for antioxidant ${ }^{17}$, antimicrobial ${ }^{17},{ }^{18}$, DNA gyrase inhibitor ${ }^{18,19}$, antitumor ${ }^{20}$ and antiinflammatory ${ }^{21,} 22$ properties.

Besides medicinal applications chalcones have a valuable place in the synthetic organic chemistry. These can serve as a key synthon for various heterocycles like flavones, pyrazolines and aurones. Flavones are known for their antimicrobial ${ }^{23}$, antiviral ${ }^{24}$, anticance ${ }^{25}$ and anti- inflammatory ${ }^{26}$ properties. Literature data also revealed that chromones substituted with pyrazole nucleus exhibit antimicrobial ${ }^{27,28}$ activities.

Pyrazolines are interesting five membered heterocyclic compounds which are associated with varied bioactivities ${ }^{29},{ }^{30}$. Recently pyrazole and thiazole based pyrazolines have emerged as an attractive lead compounds in drug development due to their antimicrobial activity profile ${ }^{31-34}$.<smiles>NC(=O)c1[nH]nc(C2OC(CO)C(O)C2O)c1O</smiles>

Pyrazofurin (antimicrobial)<smiles>Cc1cc(=O)n(-c2ccccc2)n1C</smiles>

Antipyrine (anti-inflammatory)<smiles>COC1C(C)(C)OC2(C)Oc3ccc4c(=O)cc(-c5ccccc5)oc4c3C12C</smiles>

Semiglabrin (Antifungal)
Aurones are less studied flavonoids and can be developed into a potential class of bioactive compounds. These have been reported to possess antimicrobial ${ }^{35}$, antioxidant ${ }^{36,37}$, anticancer ${ }^{38}$ and anti-inflammatory ${ }^{39}$ activities.

Introduction of fluorine in heterocycles is an attractive strategy in developing potential therapeutics. High electronegativity and small size of fluorine can offer noticeable changes in bioactivities of a molecule. Much of the current research is concentrated towards fluorinated compounds due to their broad bioactivity spectrum $^{40-43}$.

In focus of this literature survey we have synthesized fluorine containing thiazolyl pyrazole based heterocycles.

\section{EXPERIMENTAL}

The melting points were determined by open capillary method and are uncorrected. IR spectra were recorded in $\mathrm{KBr}$ on IRAffinity-1 Fourier transform infrared spectrophotometer (Shimadzu). ${ }^{1} \mathrm{H}$ NMR spectra were recorded on Bruker Avance II $400 \mathrm{MHz}$ NMR spectrometer using TMS as an internal standard and DMSO- $d_{6}$ as a solvent. The mass spectra were recorded on HP 1100 LC/MSD instrument.

1-(2-Hydroxyphenyl)-3-[3-(2,4-dimethyl-thiazol-5yl)-1-(4-fluoro-phenyl)-1 H-pyrazol-4-yl]propenone 3a-f

A mixture of 2-hydroxyacetophenone 1 $(0.02 \mathrm{~mol})$ and aldehyde 2 was dissolved in $30 \mathrm{~mL}$ of ethanol and $15 \mathrm{~mL}$ of $50 \% \mathrm{KOH}$ solution. The reaction mixture was stirred at room temperature for $48 \mathrm{~h}$. The progress of the reaction was monitored by TLC. After completion of the reaction, the contents were poured over crushed ice with acetic acid. The solid product thus obtained was filtered and crystallized from ethanol to get compound 3a-f. Spectral data of 3a-f compounds is given below.

1-(5-Chloro-2-hydroxy-4-methylphenyl)-3-[3-(2,4dimethyl-thiazol-5-yl)-1-(4-fluoro-phenyl)-1Hpyrazol-4-yl]-propenone (3a)

IR (KBr): 3151, 2959, 1659, 1565, 1155, 1026, 835, $742 \mathrm{~cm}^{-1} ;{ }^{1} \mathrm{H}$ NMR (DMSO- $d_{6}$ ): $\delta 2.34$ (s, $3 \mathrm{H}), 2.42(\mathrm{~s}, 3 \mathrm{H}), 2.71(\mathrm{~s}, 3 \mathrm{H}), 6.98(\mathrm{~s}, 1 \mathrm{H}), 7.37-$ $7.41(\mathrm{t}, 2 \mathrm{H}, \mathrm{J}=8.7 \mathrm{~Hz}), 7.69(\mathrm{~d}, 1 \mathrm{H}, \mathrm{J}=15 \mathrm{~Hz}), 7.84$ $(\mathrm{d}, 1 \mathrm{H}, \mathrm{J}=15 \mathrm{~Hz}), 7.92-7.95(\mathrm{~m}, 2 \mathrm{H}), 8.08(\mathrm{~s}, 1 \mathrm{H})$, $9.40(\mathrm{~s}, 1 \mathrm{H}), 12.60(\mathrm{~s}, 1 \mathrm{H})$. 
1-(3,5-Dichloro-2-hydroxy-phenyl)-3-[3-(2,4dimethyl-thiazol-5-yl)-1-(4-fluoro-phenyl)-1Hpyrazol-4-yl]-propenone (3b)

IR (KBr): 3151, 2959, 1659, 1565, 1155, 1026, 835, $742 \mathrm{~cm}^{-1} ;{ }^{1} \mathrm{H}$ NMR (DMSO- $d_{6}$ ): $\delta 2.41$ (s, $3 \mathrm{H}), 2.70(\mathrm{~s}, 3 \mathrm{H}), 7.47(\mathrm{t}, 2 \mathrm{H}, \mathrm{J}=8.8 \mathrm{~Hz}$ ), $7.71(\mathrm{~d}$, $1 \mathrm{H}, \mathrm{J}=15.2 \mathrm{~Hz}), 7.87-7.95(\mathrm{~m}, 4 \mathrm{H}), 8.15(\mathrm{~d}, 1 \mathrm{H}, \mathrm{J}=$ $2.4 \mathrm{~Hz}), 9.45(\mathrm{~s}, \mathrm{H}), 13.19$ (s, 1H): MS: $\mathrm{M}^{+} 488$.

1-(5-Chloro-2-hydroxyphenyl)-3-[3-(2,4-dimethylthiazol-5-yl)-1-(4-fluoro-phenyl)-1H-pyrazol-4-yl]propenone (3c)

IR (KBr): 3157, 1654, 1573, 1141, 1010, $867,725 \mathrm{~cm}^{-1} ;{ }^{1} \mathrm{H}$ NMR (DMSO- $\left.d_{6}\right): \delta 2.48(\mathrm{~s}, 3 \mathrm{H}$ ), $2.69(\mathrm{~s}, 3 \mathrm{H}), 6.94(\mathrm{~d}, 1 \mathrm{H}, \mathrm{J}=8 \mathrm{~Hz}), 7.15-7.30(\mathrm{~m}$, $3 \mathrm{H})$, 7.52-7.79 (m, 2H), 7.86-7.91 (m, 2H), $8.09(\mathrm{~d}$, $1 \mathrm{H}, \mathrm{J}=2 \mathrm{~Hz}), 9.06(\mathrm{~s}, 1 \mathrm{H}), 12.24(\mathrm{~s}, 1 \mathrm{H})$.
1-(3-Chloro-6-hydroxy-2,4-dimethylphenyl)-3-[3(2,4-dimethyl-thiazol-5-yl)-1-(4-fluoro-phenyl)-1Hpyrazol-4-yl]-propenone (3d)

IR (KBr): 3215, 1649, 1575, 1155, 1006, 835, $722 \mathrm{~cm}^{-1} ;{ }^{1} \mathrm{H}$ NMR (DMSO- $d_{6}$ ): $\delta 2.13$ (s, 3H), 2.30 \& 2.32 (two singlets, $6 \mathrm{H}$ ), 2.65 (s, 3H), 6.72 (s $1 \mathrm{H}), 6.83(\mathrm{~d}, 1 \mathrm{H}, \mathrm{J}=16 \mathrm{~Hz}), 7.10(\mathrm{~d}, 1 \mathrm{H}, \mathrm{J}=16 \mathrm{~Hz})$, $7.29(\mathrm{t}, 2 \mathrm{H}, \mathrm{J}=8.8 \mathrm{~Hz}), 7.88-7.92(\mathrm{~m}, 2 \mathrm{H}), 9.14(\mathrm{~s}$, $1 \mathrm{H}), 12.48(\mathrm{~s}, 1 \mathrm{H})$.

3-[3-(2,4-Dimethyl-thiazol-5-yl)-1-(4fluorophenyl)-1H-pyrazol-4-yl]-1-(2-hydroxy-5methylphenyl)-propenone (3e)

IR (KBr): 3192, 1650, 1546, 1161, 1033, $837 \mathrm{~cm}^{-1} ;{ }^{1} \mathrm{H}$ NMR (DMSO- $d_{6}$ ): $\delta 2.35$ (s, 3H), 2.42 $(\mathrm{s}, 3 \mathrm{H}), 2.71(\mathrm{~s}, 3 \mathrm{H}), 6.88(\mathrm{~d}, 1 \mathrm{H}, \quad \mathrm{J}=8 \mathrm{~Hz}), 7.31-$ $7.41(\mathrm{~m}, 4 \mathrm{H}), 7.68(\mathrm{~d}, 1 \mathrm{H}, \mathrm{J}=15 \mathrm{~Hz}), 7.83-7.95(\mathrm{~m}$, $3 \mathrm{H}), 9.37(\mathrm{~s}, 1 \mathrm{H}), 12.46(\mathrm{~s}, 1 \mathrm{H})$.<smiles>[R]c1c([R])c(O)c(C(=O)/C=C/c2cn(-c3ccc(F)cc3)nc2-c2sc(C)nc2C)c(C(=O)[CH-]c2nc(C)c(-c3nn(-c4ccc(F)cc4)cc3C=O)s2)c1[R]</smiles>

Scheme 1:<smiles></smiles>

Scheme 2: 
1-(5-Bromo-2-hydroxyphenyl)-3-[3-(2,4-dimethylthiazol-5-yl)-1-(4-fluoro-phenyl)-1H-pyrazol-4-yl]propenone (3f)

IR (KBr): 3192, 1650, 1546, 1161, 1033, $837 \mathrm{~cm}^{-1} ;{ }^{1} \mathrm{H}$ NMR (DMSO- $d_{6}$ ): $\delta 2.50(\mathrm{~s}, 3 \mathrm{H}), 2.69$ (s, $3 \mathrm{H}), 6.95(\mathrm{~d}, 1 \mathrm{H}, \mathrm{J}=8 \mathrm{~Hz}), 7.17-7.31(\mathrm{~m}, 3 \mathrm{H}), 7.55-$ $7.82(\mathrm{~m}, 2 \mathrm{H}), 7.91(\mathrm{dd}, 2 \mathrm{H}, \mathrm{J}=8.9 \& 4.5 \mathrm{~Hz}), 8.17(\mathrm{~d}$, $1 \mathrm{H}, \mathrm{J}=2 \mathrm{~Hz}), 8.97(\mathrm{~s}, 1 \mathrm{H}), 12.55(\mathrm{~s}, 1 \mathrm{H})$.

2-[3-(2,4-Dimethyl-1,3-thiazol-5-yl)-1-(4fluorophenyl)-1H-pyrazol-4-yl]-4H-chromen-4one $4 a-f$

Chalcone $3(0.001 \mathrm{~mol})$ was dissolved in $15 \mathrm{~mL}$ of DMSO containing catalytic amount of iodine. The contents were heated at $140^{\circ} \mathrm{C}$ for 2.5 hrs and left overnight undisturbed. The reaction mixture was poured over crushed ice and separated solid product $\mathbf{4}$ was filtered, washed with cold water followed by $10 \%$ Sodium thiosulphate solution and again with cold water. Product was crystallized from ethanol. Spectral data of compounds $\mathbf{4 a - f}$ is given below.

6-Chloro-2-[3-(2,4-dimethyl-thiazol-5-yl)-1-(4fluorophenyl)-1H-pyrazol-4-yl]-7-methylchromen-4-one (4a)

IR (KBr):3059, 1665, 1595, 1155, 1006, $835,742 \mathrm{~cm}^{-1} ;{ }^{1} \mathrm{H}$ NMR (DMSO- $\left.d_{6}\right): \delta 2.30(\mathrm{~s}, 3 \mathrm{H}$ ), $2.44(\mathrm{~s}, 3 \mathrm{H}), 2.68(\mathrm{~s}, 3 \mathrm{H}), 6.60(\mathrm{~s}, 1 \mathrm{H}), 7.22(\mathrm{~s}, 1 \mathrm{H})$, $7.35(\mathrm{t}, 2 \mathrm{H}, \mathrm{J}=8.7 \mathrm{~Hz}), 7.95-8.01(\mathrm{~m}, 3 \mathrm{H}), 9.33(\mathrm{~s}$, $1 \mathrm{H})$.

6,8-Dichloro-2-[3-(2,4-dimethyl-thiazol-5-yl)-1-(4fluorophenyl)-1H-pyrazol-4-yl]-chromen-4-one (4b)

IR (KBr): 3059, 1665, 1595, 1155, 1006, 835, $742 \mathrm{~cm}^{-1} ;{ }^{1} \mathrm{H}$ NMR (DMSO- $d_{6}$ ): $\delta 2.36(\mathrm{~s}, 3 \mathrm{H}$ ), $2.52(\mathrm{~s}, 3 \mathrm{H}), 6.92(\mathrm{~s}, 1 \mathrm{H}), 7.48(\mathrm{t}, 2 \mathrm{H}, \mathrm{J}=8.8 \mathrm{~Hz}$ ), $7.92(\mathrm{~d}, 1 \mathrm{H}, \mathrm{J}=2.4 \mathrm{~Hz}), 7.99(\mathrm{dd}, 2 \mathrm{H}, \mathrm{J}=4.6$ \& 8.8 $\mathrm{Hz}$ ), 8.09 (d, $1 \mathrm{H}, J=2.4 \mathrm{~Hz}$ ), 9.52 (s, 1H): MS: $\mathrm{M}+2$ 488.

6-Chloro-2-[3-(2,4-dimethyl-thiazol-5-yl)-1-(4fluorophenyl)-1H-pyrazol-4-yl]-chromen-4-one (4c)

IR (KBr): 3051, 1664, 1593, 1141, 1010, $839 \mathrm{~cm}^{-1} ;{ }^{1} \mathrm{H}$ NMR (DMSO- $d_{6}$ ): $\delta 2.39$ (s, 3H), 2.70 (s, $3 \mathrm{H}), 6.69(\mathrm{~s}, 1 \mathrm{H}), 7.12(\mathrm{~d}, 1 \mathrm{H}, \mathrm{J}=8 \mathrm{~Hz}), 7.24-7.36$ $(\mathrm{m}, 2 \mathrm{H}), 7.73-7.81(\mathrm{~m}, 3 \mathrm{H}), 7.82(\mathrm{~d}, 1 \mathrm{H}, \mathrm{J}=2 \mathrm{~Hz})$, $9.42(\mathrm{~s}, 1 \mathrm{H})$.
6-Chloro-2-[3-(2,4-dimethyl-thiazol-5-yl)-1-(4fluorophenyl)-1H-pyrazol-4-yl]-5,7-dimethylchromen-4-one (4d)

IR (KBr): 3070,1662, 1592, 1156, 1006, 835, $742 \mathrm{~cm}^{1} ;{ }^{1} \mathrm{H}$ NMR (DMSO- $\left.d_{6}\right): \delta 2.34(\mathrm{~s}, 3 \mathrm{H})$, $2.36(\mathrm{~s}, 3 \mathrm{H}), 2.45(\mathrm{~s}, 3 \mathrm{H}), 2.70(\mathrm{~s}, 3 \mathrm{H}), 6.71(\mathrm{~s}, 1 \mathrm{H})$, $7.02(\mathrm{~s}, 1 \mathrm{H}), 7.27-7.36(\mathrm{~m}, 2 \mathrm{H}), 7.90-7.99(\mathrm{~m}, 2 \mathrm{H})$, $9.39(\mathrm{~s}, 1 \mathrm{H})$.

2-[3-(2,4-Dimethyl-thiazol-5-yl)-1-(4-fluorophenyl)1H-pyrazol-4-yl]-6-methyl-chromen-4-one (4e)

IR (KBr): 3062, 1668, 1586, 1159, 1033 , $837 \mathrm{~cm}^{-1} ;{ }^{1} \mathrm{H}$ NMR (DMSO- $d_{6}$ ): $\delta 2.29$ (s, 3H), 2.41 (s, $3 \mathrm{H}), 2.68(\mathrm{~s}, 1 \mathrm{H}), 6.65(\mathrm{~s}, 1 \mathrm{H}), 7.18(\mathrm{~d}, 1 \mathrm{H}, \mathrm{J}=8.2$ $\mathrm{Hz}), 7.27-7.36(\mathrm{t}, 2 \mathrm{H}, \mathrm{J}=8.9 \mathrm{~Hz}), 7.68-7.77(\mathrm{~m}, 3 \mathrm{H})$, $7.89(\mathrm{~d}, 1 \mathrm{H}, \mathrm{J}=2.1 \mathrm{~Hz}), 9.36(\mathrm{~s}, 1 \mathrm{H})$.

6-Bromo-2-[3-(2,4-dimethyl-thiazol-5-yl)-1-(4fluorophenyl)-1H-pyrazol-4-yl]-chromen-4-one (4f) IR (KBr): 3092, 1665, 1590, 1163, 1033, $837 \mathrm{~cm}^{-1} ;{ }^{1} \mathrm{H}$ NMR (DMSO- $d_{6}$ ): $\delta 2.43$ (s, 3H), 2.70 (s, $3 \mathrm{H}), 6.67(\mathrm{~s}, 1 \mathrm{H}), 7.21(\mathrm{~d}, 1 \mathrm{H}, \mathrm{J}=8.5 \mathrm{~Hz}), 7.38-7.44$ (m, 2H), 7.90 (dd, $1 \mathrm{H}, J=8 \& 2 \mathrm{~Hz}), 7.98-8.01$ (m, $2 \mathrm{H}), 8.10(\mathrm{~d}, 1 \mathrm{H}, \mathrm{J}=2 \mathrm{~Hz}), 9.43(\mathrm{~s}, 1 \mathrm{H})$.

2-[3'-(2,4-Dimethyl-thiazol-5-yl)-1'-(4-florophenyl)3,4-dihydro-2H,1'H-[3,4']bipyrazolyl-5-yl]-phenol $5 a-f$

The compound $3(0.001 \mathrm{~mol})$ was dissolved in ethanol $(15 \mathrm{~mL})$ containing $(1 \mathrm{~mL})$ hydrazine hydrate. The resulting reaction mixture was refluxed for $4 \mathrm{~h}$. To the same reaction mixture glacial acetic acid $(2 \mathrm{~mL})$ was added and heating was continued further for $4 \mathrm{~h}$. The reaction is monitored by TLC. After completion of reaction the contents were cooled to room temperature and poured over crushed ice. The solid product 5 thus obtained was filtered, and crystallized from ethanol to get pure products. Spectral data of compounds 5a-f is given below.

4-Chloro-2-[3'-(2,4-dimethyl-thiazol-5-yl)-1'-(4florophenyl)-3,4-dihydro-2H,1' $H$-[3,4']bipyrazolyl5-yl]-5-methyl-phenol (5a)

IR (KBr): 3315, 3112, 1639, 1228, $835 \mathrm{~cm}$

1; ${ }^{1} \mathrm{H}$ NMR (DMSO- $\left.d_{6}\right): \delta 2.31(\mathrm{~s}, 3 \mathrm{H}), 2.44(\mathrm{~s}, 3 \mathrm{H})$, $2.69(\mathrm{~s}, 3 \mathrm{H}), 3.18(\mathrm{dd}, \mathrm{J}=10 \& 16 \mathrm{~Hz}), 3.70(\mathrm{dd}, 1 \mathrm{H}$, $J=11 \& 16 \mathrm{~Hz}), 4.93(\mathrm{dd}, 1 \mathrm{H}, J=10 \& 11 \mathrm{~Hz}), 6.80$ (s, 1H), 7.29-7.36 (m, 2H), $7.70(\mathrm{~s}, 1 \mathrm{H}), 7.93$ (dd, $2 \mathrm{H}, \mathrm{J}=4.3 \& 9.6 \mathrm{~Hz}), 8.69(\mathrm{~s}, 1 \mathrm{H}), 11.12(\mathrm{~s}, 1 \mathrm{H})$. 
2,4-Dichloro-6-[3'-(2,4-dimethyl-thiazol-5-yl)-1' -(4florophenyl)-3,4-dihydro-2H,1'H-[3,4']bipyrazolyl5-yl]-phenol (5b)

IR (KBr): 3315, 3112, 1639, 1228, $835 \mathrm{~cm}$ 1; ${ }^{1} \mathrm{H}$ NMR (DMSO- $\left.d_{6}\right): \delta 2.45(\mathrm{~s}, 3 \mathrm{H}), 2.64(\mathrm{~s}, 3 \mathrm{H})$, 3.0-3.1 (m, 1H), 3.5-3.65 (m, 1H), $4.91(\mathrm{t}, 1 \mathrm{H}, \mathrm{J}=9.5$ $\mathrm{Hz}), 7.26-7.40(\mathrm{~m}, 4 \mathrm{H}), 7.86-7.94(\mathrm{~m}, 3 \mathrm{H}), 8.54$ (s, 1H), 11.90 (s, 1H); MS: M+502.

4-Chloro-2-[3'-(2,4-dimethyl-thiazol-5-yl)-1'-(4florophenyl)-3,4-dihydro-2H,1'H-[3,4']bipyrazolyl5-yl]-phenol (5c)

IR (KBr): 3320, 3110, 1630, 1214, $840 \mathrm{~cm}$

1; ${ }^{1} \mathrm{H}$ NMR (DMSO- $\left.d_{6}\right): \delta 2.45(\mathrm{~s}, 3 \mathrm{H}), 2.71$ (s, 3H), $3.14(\mathrm{dd}, 1 \mathrm{H}, \mathrm{J}=11 \& 16 \mathrm{~Hz}), 3.63(\mathrm{dd}, 1 \mathrm{H}, \mathrm{J}=11$ \& $16 \mathrm{~Hz}), 4.90(\mathrm{t}, 1 \mathrm{H}, \mathrm{J}=11 \mathrm{~Hz}), 6.84(\mathrm{~d}, 1 \mathrm{H}, \mathrm{J}=8.5$ $\mathrm{Hz}$ ), 7.26-7.32 (m, 4H), $7.70(\mathrm{~d}, 1 \mathrm{H}, \mathrm{J}=2 \mathrm{~Hz}), 7.86$ (dd, $2 \mathrm{H}, \mathrm{J}=4.5$ \& $9.6 \mathrm{~Hz}), 8.49(\mathrm{~s}, 1 \mathrm{H}), 11.15$ (s, $1 \mathrm{H})$.

4-Chloro-2-[3'-(2,4-dimethyl-thiazol-5-yl)-1'-(4florophenyl)-3,4-dihydro-2H,1'H-[3,4']bipyrazolyl5-yl]-3,5-dimethyl-phenol (5d)

IR (KBr): 3335, 3140, 1643, 1209, 848 $\mathrm{cm}^{-1} ;{ }^{1} \mathrm{H}$ NMR (DMSO-d $): \delta 2.25(\mathrm{~s}, 3 \mathrm{H}), 2.30$ (s, $3 \mathrm{H}), 2.48(\mathrm{~s}, 3 \mathrm{H}), 2.68(\mathrm{~s}, 3 \mathrm{H}), 2.97-3.04(\mathrm{~m}, 2 \mathrm{H})$, $4.83(\mathrm{t}, 1 \mathrm{H}, \mathrm{J}=9 \mathrm{~Hz}), 6.69(\mathrm{~s}, 1 \mathrm{H}), 6.90(\mathrm{~s}, 1 \mathrm{H}), 7.23$ $(\mathrm{t}, 2 \mathrm{H}, \mathrm{J}=8.9 \mathrm{~Hz}), 7.81$ (dd, $2 \mathrm{H}, \mathrm{J}=9 \& 4 \mathrm{~Hz}), 8.49$ (s, 1H), $9.95(\mathrm{~s}, 1 \mathrm{H})$.

2-[3'-(2,4-Dimethyl-thiazol-5-yl)-1'-(4-florophenyl)3,4-dihydro-2H,1'H-[3,4']bipyrazolyl-5-yl]-4methyl-phenol (5e)

IR (KBr): 3311, 3150, 1664, 1211, $831 \mathrm{~cm}$

1; ${ }^{1} \mathrm{H}$ NMR (DMSO- $\left.d_{6}\right): \delta 2.30(\mathrm{~s}, 3 \mathrm{H}), 2.42(\mathrm{~s}, 3 \mathrm{H})$, $2.67(\mathrm{~s}, 3 \mathrm{H}), 2.9(\mathrm{dd}, 1 \mathrm{H}, \mathrm{J}=9 \& 12 \mathrm{~Hz}), 3.6(\mathrm{dd}, 1 \mathrm{H}$, $J=9 \& 12 \mathrm{~Hz}), 5.2(\mathrm{t}, 1 \mathrm{H}, \mathrm{J}=9 \mathrm{~Hz}), 6.75(\mathrm{~s}, 1 \mathrm{H}), 6.87$ $(\mathrm{d}, 1 \mathrm{H}, \mathrm{J}=8.4 \mathrm{~Hz}), 7.16-7.30(\mathrm{~m}, 4 \mathrm{H}), 7.81-8.0(\mathrm{~m}$, $2 \mathrm{H}), 9.24(\mathrm{~s}, 1 \mathrm{H}), 11.88(\mathrm{~s}, 1 \mathrm{H})$.

4-Bromo-2-[3'-(2,4-dimethyl-thiazol-5-yl)-1'-(4florophenyl)-3,4-dihydro-2H,1'H-[3,4']bipyrazolyl5-yl]-phenol (5f)

IR (KBr): 3324, 3127, 1657, 1213, $843 \mathrm{~cm}$

1; ${ }^{1} \mathrm{H}$ NMR (DMSO- $\left.d_{6}\right): \delta 2.46(\mathrm{~s}, 3 \mathrm{H}), 2.72(\mathrm{~s}, 3 \mathrm{H})$, 3.20 (dd, $1 \mathrm{H}, \mathrm{J}=9$ \& $11 \mathrm{~Hz}), 3.65$ (dd, $1 \mathrm{H}, \mathrm{J}=9$ \& 11 $\mathrm{Hz}), 4.95(\mathrm{t}, 1 \mathrm{H}, \mathrm{J}=11 \mathrm{~Hz}), 6.84(\mathrm{~d}, 1 \mathrm{H}, \mathrm{J}=8.3 \mathrm{~Hz})$, 7.30-7.37 (m, 4H), $7.74(\mathrm{~d}, 1 \mathrm{H}, \mathrm{J}=2 \mathrm{~Hz}), 7.90$ (dd, $2 \mathrm{H}, \mathrm{J}=4.2 \& 9.6 \mathrm{~Hz}), 8.70(\mathrm{~s}, 1 \mathrm{H}), 11.20(\mathrm{~s}, 1 \mathrm{H})$.
2-[3-(2,4-Dimethylthiazol-5-yl)-1-(4-fluorophenyl)1H-pyrazol-4-methylene]-benzofuran-3-one 6a-f A mixture of chalcone $3(0.001 \mathrm{~mol})$ and mercuric acetate $(0.001 \mathrm{~mol})$ was dissolved in 10 $\mathrm{mL}$ dry pyridine. The reaction mixture was refluxed for 4-5 $\mathrm{h}$. The reaction was monitored by TLC. The reaction mixture was cooled to room temperature and poured over crushed ice and neutralized with conc. $\mathrm{HCl}$. The solid product 6 was filtered and crystallized from glacial acetic acid. Spectral data of synthesized compounds $6 a-f$ is given below.

5-Chloro-2-[3-(2.4-dimethyl-thiazol-5-yl)-1-(4fluoro-phenyl)-1H-pyrazol-4-ylmethylene]-6methyl-benzofuran-3-one (6a)

IR (KBr): 3076, 2918, 1705, 1650, 1599, 1512, 1174, 1053, $906 \mathrm{~cm}^{-1} ;{ }^{1} \mathrm{H}$ NMR (DMSO-d $d_{6}$ ): $\delta$ 2.39 (s, 3H), 2.42 (s, 3H), 2.71 (s, 3H), 6.81 (s, 1H), $6.98(\mathrm{~s}, 1 \mathrm{H}), 7.32-7.38(\mathrm{~m}, 2 \mathrm{H}), 7.81-7.90(\mathrm{~m}, 3 \mathrm{H})$, $8.89(\mathrm{~s}, 1 \mathrm{H})$.

5,7-Dichloro-2-[3-(2.4-dimethyl-thiazol-5-yl)-1-(4fluoro-phenyl)-1H-pyrazol-4-ylmethylene]benzofuran-3-one (6b)

IR (KBr): 3064, 2918, 1707, 1650, 1597, 1512, 1174, 833, $750 \mathrm{~cm}^{-1} ;{ }^{1} \mathrm{H}$ NMR (DMSO- $d_{6}$ ): $\delta$ $2.43(\mathrm{~s}, 3 \mathrm{H}), 2.71(\mathrm{~s}, 3 \mathrm{H}), 6.83(\mathrm{~s}, 1 \mathrm{H}), 7.38(\mathrm{t}, 2 \mathrm{H}, \mathrm{J}$ $=9 \mathrm{~Hz}), 7.79(\mathrm{~d}, 1 \mathrm{H}, \mathrm{J}=2 \mathrm{~Hz}), 7.91(\mathrm{dd}, 2 \mathrm{H}, \mathrm{J}=9$ \& $4.6 \mathrm{~Hz}), 7.98(\mathrm{~d}, 1 \mathrm{H}, \mathrm{J}=2 \mathrm{~Hz}), 8.83(\mathrm{~s}, 1 \mathrm{H}): \mathrm{MS}: \mathrm{M}+$ 486.

5-Chloro-2-[3-(2.4-dimethyl-thiazol-5-yl)-1-(4fluoro-phenyl)-1 $\mathrm{H}$-pyrazol-4-ylmethylene]benzofuran-3-one (6c). IR (KBr): 3067, 2935, 1707, 1653, 1596, 1170, 1061, $915 \mathrm{~cm}^{-1} ;{ }^{1} \mathrm{H}$ NMR (DMSO$\left.d_{6}\right): \delta 2.39(\mathrm{~s}, 3 \mathrm{H}), 2.70(\mathrm{~s}, 3 \mathrm{H}), 6.70(\mathrm{~s}, 1 \mathrm{H}), 7.29-$ $7.32(\mathrm{~m}, 4 \mathrm{H}), 7.83-7.92(\mathrm{~m}, 3 \mathrm{H}), 9.12(\mathrm{~s}, 1 \mathrm{H})$.

5-Chloro-2-[3-(2.4-dimethyl-thiazol-5-yl)-1-(4fluoro-phenyl)-1H-pyrazol-4-ylmethylene]-4,6dimethyl-benzofuran-3-one (6d)

IR (KBr): 3056, 2967, 1708, 1652, 1593, 1067, $948 \mathrm{~cm}^{-1} ;{ }^{1} \mathrm{H}$ NMR (DMSO-d $)$ ): $\delta 2.42(\mathrm{~s}, 3 \mathrm{H}$ ), $2.64(\mathrm{~s}, 3 \mathrm{H}), 2.70(\mathrm{~s}, 3 \mathrm{H}), 2.71(\mathrm{~s}, 3 \mathrm{H}), 6.61(\mathrm{~s}, 1 \mathrm{H})$, 7.36-7.43 (m, 3H), 8.0-8.04 (m, 2H), $9.02(\mathrm{~s}, 1 \mathrm{H})$.

5-Chloro-2-[3-(2.4-dimethyl-thiazol-5-yl)-1-(4fluoro-phenyl)-1H-pyrazol-4-ylmethylene]-6methyl-benzofuran-3-one (6e)

IR (KBr): 3061, 2965, 1709, 1654, 1598, 
1060, $948 \mathrm{~cm}^{-1} ;{ }^{1} \mathrm{H}$ NMR (DMSO- $\left.d_{6}\right): \delta 2.40$ (s, 3H), $2.43(\mathrm{~s}, 3 \mathrm{H}), 2.69(\mathrm{~s}, 3 \mathrm{H}), 6.71(\mathrm{~s}, 1 \mathrm{H}), 7.26-7.36(\mathrm{~m}$, $4 \mathrm{H})$, 7.86-7.96 (m, 3H), $9.03(\mathrm{~s}, 1 \mathrm{H})$.

5-Chloro-2-[3-(2.4-dimethyl-thiazol-5-yl)-1-(4fluoro-phenyl)-1H-pyrazol-4-ylmethylene]-6bromo-benzofuran-3-one (6f)

IR (KBr): 3056, 2961, 1709, 1652, 1583, 1067, 943, $726 \mathrm{~cm}^{-1} ;{ }^{1} \mathrm{H}$ NMR (DMSO- $\left.d_{6}\right): \delta 2.41$ (s, $3 \mathrm{H}), 2.71(\mathrm{~s}, 3 \mathrm{H}), 6.71(\mathrm{~s}, 1 \mathrm{H}), 7.31-7.48(\mathrm{~m}, 4 \mathrm{H})$, 7.70-8.01 (m, 3H), $9.07(\mathrm{~s}, 1 \mathrm{H})$.

\section{RESULTS AND DISCUSSION}

Synthesis of starting compounds 3a-f was carried out by known literature method as shown in scheme 1 and used as starting material for target compounds. The IR spectrum of compound $\mathbf{3 b}$ showed the bands at 1637 and $1562 \mathrm{~cm}^{-1}$ for conjugated $\mathrm{C}=\mathrm{O}$ and $\mathrm{C}=\mathrm{C}$ bonds respectively. The
${ }^{1} \mathrm{H}$ NMR spectrum of this compound showed a singlet at $\delta 13.19$ which shows the presence of $\mathrm{O}-\mathrm{H}$ proton. The signal at $\delta 9.45$ shows the presence of pyrazole ring proton. Compounds 3a-f on refluxing in DMSO containing catalytic amount of iodine were cyclized to chromones 4a-f having hetero substituent at 2-position. The IR spectrum of compound 4b showed a band at 1669, 1651 and $1608 \mathrm{~cm}^{-1}$ corresponding to $\mathrm{C}=\mathrm{O}$, aliphatic $\mathrm{C}=\mathrm{C}$ and aromatic $\mathrm{C}=\mathrm{C}$ bonds respectively. Formation of chromones is well explained by ${ }^{1} \mathrm{H}$ NMR spectrum. Absence of downfield signal at $\delta 13.19$ depicted the absence of $\mathrm{O}-\mathrm{H}$ while the singlet at 6.89 is due to proton at 3-position of chromone. Chalcone 3a-f are transformed into 2-[3'-(2,4-dimethyl-thiazol-5yl)-1'-(4-florophenyl)-3,4-dihydro- $2 \mathrm{H}, 1^{\prime} \mathrm{H}$ [3,4']bipyrazolyl-5-yl]-phenols by reacting them with hydrazine hydrate in ethanol. Formation of pyrazolines $\mathbf{5 a - f}$ is confirmed by spectral techniques. IR spectrum of compound $\mathbf{5 b}$ showed the sharp $\mathrm{N}$ -

Table 1: Characterization data of synthesized compounds

\begin{tabular}{lcccccc}
\hline Compound & $\mathbf{R}_{\mathbf{1}}$ & $\mathbf{R}_{\mathbf{2}}$ & $\mathbf{R}_{\mathbf{3}}$ & $\mathbf{R}_{\mathbf{4}}$ & M.P. $\left({ }^{\circ} \mathbf{C}\right)$ & Yield (\%) \\
\hline $3 \mathrm{a}$ & $\mathrm{H}$ & $\mathrm{Me}$ & $\mathrm{Cl}$ & $\mathrm{H}$ & $196-98$ & 56 \\
3b & $\mathrm{Cl}$ & $\mathrm{H}$ & $\mathrm{Cl}$ & $\mathrm{H}$ & $248-50$ & 59 \\
$3 \mathrm{c}$ & $\mathrm{H}$ & $\mathrm{H}$ & $\mathrm{Cl}$ & $\mathrm{H}$ & $226-28$ & 55 \\
3d & $\mathrm{H}$ & $\mathrm{Me}$ & $\mathrm{Cl}$ & $\mathrm{Me}$ & $236-38$ & 48 \\
$3 \mathrm{e}$ & $\mathrm{H}$ & $\mathrm{H}$ & $\mathrm{Me}$ & $\mathrm{H}$ & $146-48$ & 55 \\
3f & $\mathrm{H}$ & $\mathrm{H}$ & $\mathrm{Br}$ & $\mathrm{H}$ & $188-90$ & 62 \\
$4 \mathrm{a}$ & $\mathrm{H}$ & $\mathrm{Me}$ & $\mathrm{Cl}$ & $\mathrm{H}$ & $200-02$ & 62 \\
$4 \mathrm{~b}$ & $\mathrm{Cl}$ & $\mathrm{H}$ & $\mathrm{Cl}$ & $\mathrm{H}$ & $258-60$ & 64 \\
$4 \mathrm{C}$ & $\mathrm{H}$ & $\mathrm{H}$ & $\mathrm{Cl}$ & $\mathrm{H}$ & $198-200$ & 52 \\
$4 \mathrm{~d}$ & $\mathrm{H}$ & $\mathrm{Me}$ & $\mathrm{Cl}$ & $\mathrm{Me}$ & $228-30$ & 64 \\
$4 \mathrm{e}$ & $\mathrm{H}$ & $\mathrm{H}$ & $\mathrm{Me}$ & $\mathrm{H}$ & $232-34$ & 56 \\
$4 \mathrm{f}$ & $\mathrm{H}$ & $\mathrm{H}$ & $\mathrm{Br}$ & $\mathrm{H}$ & $268-70$ & 58 \\
$5 \mathrm{a}$ & $\mathrm{H}$ & $\mathrm{Me}$ & $\mathrm{Cl}$ & $\mathrm{H}$ & $200-02$ & 42 \\
$5 \mathrm{~b}$ & $\mathrm{Cl}$ & $\mathrm{H}$ & $\mathrm{Cl}$ & $\mathrm{H}$ & $196-98$ & 54 \\
$5 \mathrm{c}$ & $\mathrm{H}$ & $\mathrm{H}$ & $\mathrm{Cl}$ & $\mathrm{H}$ & $180-82$ & 49 \\
$5 \mathrm{~d}$ & $\mathrm{H}$ & $\mathrm{Me}$ & $\mathrm{Cl}$ & $\mathrm{Me}$ & $184-86$ & 54 \\
$5 \mathrm{e}$ & $\mathrm{H}$ & $\mathrm{H}$ & $\mathrm{Me}$ & $\mathrm{H}$ & $200-02$ & 46 \\
$5 f$ & $\mathrm{H}$ & $\mathrm{H}$ & $\mathrm{Br}$ & $\mathrm{H}$ & $194-96$ & 48 \\
$6 \mathrm{a}$ & $\mathrm{H}$ & $\mathrm{Me}$ & $\mathrm{Cl}$ & $\mathrm{H}$ & $170-72$ & 62 \\
$6 \mathrm{~b}$ & $\mathrm{Cl}$ & $\mathrm{H}$ & $\mathrm{Cl}$ & $\mathrm{H}$ & $240-42$ & 68 \\
$6 \mathrm{c}$ & $\mathrm{H}$ & $\mathrm{H}$ & $\mathrm{Cl}$ & $\mathrm{H}$ & $242-44$ & 62 \\
$6 \mathrm{~d}$ & $\mathrm{H}$ & $\mathrm{Me}$ & $\mathrm{Cl}$ & $\mathrm{Me}$ & $188-90$ & 64 \\
$6 \mathrm{e}$ & $\mathrm{H}$ & $\mathrm{H}$ & $\mathrm{Me}$ & $\mathrm{H}$ & $228-30$ & 66 \\
$6 f$ & $\mathrm{H}$ & $\mathrm{H}$ & $\mathrm{Br}$ & $\mathrm{H}$ & $170-72$ & 68 \\
\hline & & & & & & \\
\hline & & & & & & \\
\hline
\end{tabular}


Table 2: Antibacterial activity

\begin{tabular}{lcccc}
\hline Compound & \multicolumn{5}{c}{ Zone of inhibition in mm at concentration $\mathbf{1 0 0 ~} \mathrm{\mu g} / \mathrm{mL}$} \\
& B. subtilis & S. aureus & E. coli & S. typhi \\
\hline $5 \mathrm{a}$ & - & - & - & - \\
$5 \mathrm{~b}$ & 16 & - & - & - \\
$5 \mathrm{c}$ & - & 15 & 15 & - \\
$5 \mathrm{~d}$ & 14 & 15 & 13 & - \\
$5 \mathrm{e}$ & 13 & 15 & - & - \\
$5 \mathrm{f}$ & 16 & 15 & 12 & - \\
$6 \mathrm{a}$ & 18 & 17 & 14 & - \\
$6 \mathrm{~b}$ & 14 & 15 & 14 & - \\
$6 \mathrm{c}$ & 14 & 15 & 13 & - \\
$6 \mathrm{~d}$ & 12 & 14 & 16 & - \\
$6 \mathrm{e}$ & weak & 12 & 12 & - \\
$6 f$ & 13 & 13 & 12 & - \\
Ciprofloxacin & 35 & 46 & 40 & 39 \\
\hline
\end{tabular}

$\mathrm{H}$ band at $3336 \mathrm{~cm}^{-1}$ and absence of band in carbonyl region supports the formation of pyrazolines ring. The ${ }^{1} \mathrm{H}$ NMR spectrum showed the multiplets at $3.19,3.65$ confirm the presence of diastereotopic methylene protons in pyrazoline ring while triplet at 4.91 is due to methane proton of pyrazolines ring. Compounds 3a-f were cyclized to 2-[3-(2,4-dimethylthiazol-5-yl)-1-(4-fluorophenyl)$1 \mathrm{H}$-pyrazol-4-methylene]-benzofuran-3-one 6a-f with mercuric acetate in dry pyridine. The IR spectrum of compound $\mathbf{6 b}$ exhibited a band at 1707 $\mathrm{cm}^{-1}$ which shows the presence of conjugated carbonyl group in a strained five membered ring. The ${ }^{1} \mathrm{H}$ NMR spectrum of $6 \mathbf{b}$ showed the singlet at 6.83 corresponding to a olefinic proton in a $\alpha, \beta-$ unsaturated enone system. Absence of $\mathrm{O}-\mathrm{H}$ proton peak also supports the formation of the compounds 6a-f. The formation of all the newly synthesized compounds is confirmed by mass spectrometry also.

\section{Antimicrobial activity}

The antimicrobial activity of synthesized compounds was determined in vitro against four bacterial strains. For this study, the test cultures of bacterial strains Escherichia coli, Salmonella typhi, Bacillus subtilis and Staphylococcus aureus were maintained in nutrient agar slants at $37^{\circ} \mathrm{C}$. The antbacterial activity of compounds against test bacteria was determined by agar well diffusion method using standard antibiotic ciprofloxacin as positive control and DMSO as negative control. All the experiments were performed in triplicate. The results of this investigation showed that compounds 3a-f and $\mathbf{4 a - f}$ are inactive against all the test organisms at $100 \mu \mathrm{g} / \mathrm{mL}$ concentration.

\section{ACKNOWLEDGEMENTS}

The authors are thankful to Management of Ahmednagar Jilha Maratha Vidya Prasarak Samaj, Ahmednagar for constant encouragement and providing necessary research facilities. The authors are also thankful to the Director, SAIF, Punjab University, Chandigarh for providing spectral information.

\section{REFERENCES}

1. Rostom, S. A. F.; Bioorg. Med. Chem. 2010, 18, 2767-2776.

2. Khloya, P.; Kumar, P.; Mittal, A.; Aggarwal, N. K.;
Sharma, P.K. Org.Med.Chem.Lett. 2013, http:/ /www.orgmedchemlett.com/content/3/1/9.

3. Pattan, S. R.; Patel, P. V.; Athare, G. S.; Jagnar, 
A. B.; Nirmal, S. A.; Pattan, J. S. Bulgarian Chem. Commun. 2014, 46, 125-134.

4. Dai, H.; Xiao, Y-S.; Li, Z.; Xu, X-Y.; Qian, X-H. Chinese Chem. Lett. 2014, 25, 1014-1016.

5. Jaishree, V.; Ramdas, N.; Sachin, J.; Ramesh, B. J. Saudi Chem. Soc. 2012, 16, 371-376.

6. Karegoudar, P.; Karthikeyan, M. S.; Prasad, D. J.; Mahalinga, M.; Holla, B. S.; Kumari, N. S. Eur. J. Med. Chem. 2008, 43, 261-267.

7. Mhaske, P. C.; Vadgaonkar, K. S.; Jadhav, R. P.; Bobade, V. D. J. Korean Chem. Soc. 2011, 55(5), 882-886.

8. Mhaske, P. C.; Shelke, S. H.; Raundal, H. N.; Jadhav, R. P. J. Korean Chem. Soc. 2014, 58(1), 62-67.

9. Luzina, E. L.; Popov, A. V. Eur. J. Med. Chem. 2009, 44, 4944-4953.

10. Sachdeva, H.; Dwivedi, D.; Goyal, P. Org. Chem. International 2013, http://dx.doi.org./ 10.1155/2013/976032.

11. Bandock, S.; Fadaly, W.; Metwally, M. A. Eur. J. Med. Chem. 2010, 45, 3692-3701.

12. Desai, N. C.; Joshi, V. V.; Rajpara, K. M.; Vaghani, H. V.; Satodiya, H. M. J. Fluorine Chem. 2012, 142, 67-78.

13. Liaras, K.; Geronikaki, A.; Glamoclija, J.; Ciric, A.; Sokovic, M. Bioorg. Med. Chem. 2011, 19, 3135-3140.

14. Lin, Y-M.; Zhou, Y.; Flavin, M. T.; Zhou, L-M.; Nie, W.; Chen, F-C. Bioorg. Med. Chem. 2002, 10, 2795-2802.

15. Alam, S.; Panda, R.; Kachroo, M. Indian J. Chem. 2014, 53B, 440-443.

16. Cabrera, M.; Simoens, M.; Falchi, G.; Lavaggi, M. L.; Piro, O. E.; Castellano, E. E.; Vidal, A.; Azqueta, A.; Monge, A.; de Cerain, A. L.; Sagrera, G.; Seoane, G.; Cerecetto H.; Gonzalez, M. Bioorg. Med. Chem. 2007, 15, 3356-3367.

17. Doan, T. N.; Tran, D. T. Pharmacol. Pharma. 2011, 2, 282-288.

18. Tanitame, A.; Oyamada, Y.; Ofuji, K.; Fujimoto, M.; Iwai, N.; Hiyama, Y.; Suzuki, K.; Ito, H.; Terauchi, H.; Kawasako, M.; Nagai, K.; Wachi, M.; Yamagishi, J. J. Med. Chem. 2004, 47, 3693-3696.

19. Tanitame, A.; Oyamada, Y.; Ofuji, K.; Terauchi, H.; Motoji, K.; Wachi,; Yamagishi, J. Bioorg. Med. Chem. Lett. 2005, 15, 4299-4303.

20. Insuasty, B.; Tigreos, A.; Orozco, F.; Quiroga,
J.; Abonia, R.; Nogueras, M.; Sanchez, A.; Cobo, J. Bioorg. Med. Chem. 2010, 18, 49654974.

21. Sharma P. K.; Kumar, S.; Kumar, P.; Kaushik, P.; Kaushik, D.; Dhingra, Y.; Aneja, K. R. Eur.J. Med. Chem. 2010, 45, 2650-2655.

22. Bandgar, B. P.; Gawande, S. S.; Bodade, R. G.; Gawande, N. M.; Khobragade, C. N. Bioorg. Med. Chem. 2009, 17, 8168-8173.

23. Salem, M. S.; Marzouk, M. I.; Ali, S. N.; Madkour, H. M. F. Eur. J. Chem. 2012, 2(2), 220-227.

24. Chung, S-T.; Chien, P-Y.; Huang, W-H.; Yao, C-W.; Lee, A-R. Chem. Pharma. Bull. 2014, 62(5), 415-421.

25. Bao, J.; Luo, J-F.; Qin, X-C.; Xu, X-Y.; Xu, X-Y.; Zhang, X-Y.; Tu, Z-C.; Qi, S-H. Bioorg. Med. Chem. Lett. 2014, 24, 2433-2436.

26. Bano, S.; Javed, K.; Ahmed, S.; Ratish, I. G.; Singh, S.; Chaitanya, M.; Arunasree, K. M. Alam, M. S. Eur. J. Med. Chem. 2013, 65, 5159.

27. Prakash, O.; Kumar, R.; Sherawat, R. Eur. J. Med. Chem. 2009, 44, 1763-1767.

28. Gadhave, A. G.; Burungale, A. S.; Kuchekar, S.; Karale, B. K. Indian J. Heterocycl. Chem. 2012, 22, 191-196.

29. Abdalla, M. M.; Al-Omar, M. A.; Bhat, M. A.; Amr, A-G. E; Al-Mohizea, A. M. International J. Biol. Macromol. 2012, 50, 1127-1132.

30. Bandgar, B. P.; Adsul, L. K.; Chavan, H. V.; Jalde, S. S.; Shringare, S. N.; Shaikh, R.; Meshram, R. J.; Gacche, R. N.; Masnad, V. Bioorg. Med. Chem. Lett. 2012, 22, 58395844.

31. Akolkar, H. N.; Karale, B. K. Indian J. Heterocycl. Chem. 2013, 22, 319-320.

32. Girisha, K. S.; Kalluraya, B.; Narayana, V.; Padmashree, Eur. J. Med. Chem. 2010, 45, 4640-4644.

33. Dawane, B. S.; Konda, S. G.; Mandawad, G. G.; Shaikh, B. M. Eur. J. Med. Chem. 2010, 45, 387-392.

34. Sharifzadeh, B.; Mahmoodi, N. O.; Mamaghani, M.; Tabatabaeian, K.; Chirani, A. S.; Nikokar, I. Bioorg. Med. Chem. Lett. 2013, 23, 548-551.

35. Bandgar, B. P.; Patil, S. A.; Korbad, B. L.; Biradar, S. C.; Nile, S. N.; Khobragade, C. N. Eur. J. Med. Chem. 2010, 45, 3223-3227. 
36. Jardosh, H. H.; Patel, M. P. Arabian J. Chem. 2014, htpp://dx.doi.org/10.1016/ j.arabjc.2014.05.014.

37. Detsi, A.; Majdalani, M.; Kontogiorgis, C. A.; Hadjipavlou-Litina, D.; Kefalas, P. Bioorg. Med. Chem. Lett. 2009, 17, 8073-8085.

38. Manjulatha, K.; Srinivas, S.; Mulakayale, N.; Rambhau, D.; Prabhakar, M.; Arunasree, K. M.; Alvia, M.; Rao, M. V. B.; Pal, M. Bioorg. Med. Chem. Lett. 2012, 22, 6160-6165.

39. Shin, S. Y.; Shin, M. C.; Shin, J. S.; Lee, K. T.; Lee, Y. S. Bioorg. Med. Chem. Lett. 2011, 21, 4520-4523.
40. Shelke, S.; Salunkhe, N.; Sangale, S.; Bhalerao, S.; Naik, N.; Mhaske, G.; Jadhav, R.; Karale, B. K. J. Korean Chem. Soc. 2010, 54(1), 59-64.

41. Gadakh, A. V.; Pandit, C.; Rindhe, S. S.; Karale, B. K. Bioorg. Med. Chem. Lett. 2010, 20, 55725576.

42. Patel, V. M.; Desai, K. R. Indian J. Chem. 2005, 44B, 1084-1087.

43. Hammam, A. E. G.; Salam, O. I. A.; Mohamaed, A. M.; Hafez, N. A. Indian J. Chem. 2005, 44B, 1887-1893. 